\title{
Association of Abnormal Liver Function Tests to Outcomes in Patients with a New Diagnosis of Heart Failure with Reduced Ejection Fraction in the Outpatient Clinic
}

Koganti $\mathbf{S}^{1}$, Dasgupta $\mathbf{S}^{1}$, Crossman $\mathbf{R}^{1}$ and Banerjee $\mathbf{P}^{1,2 *}$

${ }^{1}$ Department of Cardiology, University Hospitals of Coventry and Warwickshire, Coventry, England, UK

${ }^{2}$ Faculty of Health \& Life Sciences, Coventry University, Coventry, England, UK

\begin{abstract}
Aim: To evaluate the relationship of abnormal Liver Function Tests (LFTs) to outcomes in patients with heart failure due to reduced ejection fraction (HFREF) at the time of diagnosis in the heart failure clinic.

Methods: We undertook a retrospective analysis of all patients diagnosed with HFREF in the heart failure outpatient clinic of a tertiary teaching hospital from 2007 to 2011. Ninety-five patients with abnormal LFTs at presentation were compared with 127 contemporaneous randomly chosen patients with normal LFTs and their outcomes were analyzed. Time to event data mortality and admission rates, as well as length of hospital stay during admissions were assessed.
\end{abstract}

Results: Of 222 patients $69 \%$ were Male. The mean age across both the groups was $69.2 \pm 13.2$ years. The major ethnic group was of white background (78\%). Majority of patients were in NYHA class $2(54.5 \%)$. On multivariate analysis, low albumin at the time of diagnosis was the strongest predictor of all cause death (OR $1.24,95 \% \mathrm{Cl} 1.15-1.35, \mathrm{p}<0.0001)$. Patients in the abnormal LFT group had a longer in-hospital stay compared to those in the normal LFT group ( $25.9 \pm 29.1$ days vs. $17.2 \pm 20.5$ days, $p=0.01)$. Low albumin and elevated alkaline phosphatase (ALP) were the strongest predictors of increased length of in-hospital stay following heart failure related admissions on multivariate analysis (OR $-0.583, \mathrm{Cl}-1.119,-0.046, \mathrm{p}=0.035$ and $\mathrm{OR} 0.033, \mathrm{Cl} 0.005,0.061$, $p=0.02$, respectively). Every unit increase of albumin resulted in reduction of in hospital stay by 0.875 days. Time to event analysis showed more deaths in the abnormal LFT group when compared to the normal LFT group (log rank test $p=0.012$ ). Although an elevated ALP was seen to be associated with readmission, multivariate analysis found this to be a trend only, with none of the LFTs being predictive.

Conclusion: Abnormal LFTs in the outpatient setting in patients with HFREF was associated with higher mortality in this study. Low Albumin was independently associated with mortality and length of hospital stay due to heart failure. An elevated alkaline phosphatase also seems to be important in predicting length of stay in hospital. Triaging acute heart failure patients due to Left Ventricular Systolic Dysfunction (LVSD) using abnormal LFTs as biomarkers for more intensive treatment and monitoring would seem advisable. However, this is a retrospective analysis of a relatively small number of patients and the conclusions must be seen in that light.

Keywords: Heart failure; Liver function tests; Mortality; Readmissions; Length of hospital stay

Abbreviations: NYHA: New York Heart Association; EF: Ejection Fraction; CAD: Coronary Artery Disease; ACE-I: Angiotensin Converting Enzyme Inhibitor; ALT: Alanine Aminotransferase; ALP: Alkaline Phosphatase; NS: P Value Not Statistically Significant

\section{Introduction}

There are 900,000 people with symptomatic heart failure in the UK [1]. Although there is a trend towards improvement of prognosis of heart failure patients, mortality still remains high, particularly so in the first year [2-4]. This is despite of advances in treating heart failure with therapies ranging from optimal medical therapy to invasive therapies such as Cardiac Resynchronization Therapy (CRT). Thus, ongoing research into avenues to identify patients at greater risk of mortality early on is justified. One such avenue is the prognostic role of abnormal liver function tests (LFTs) in patients with new diagnosis of heart failure with reduced ejection fraction (HFREF). Abnormal LFTs, predominantly alkaline phosphatase (ALP) and bilirubin, were noted to occur in patients with right heart failure and tricuspid regurgitation as a result of hepatic congestion [5]. Likewise, abnormal aminotransferases are seen as a result of ischaemic hepatitis secondary to cardiogenic shock [6]. Low albumin reflecting poor nutrition is often seen in patients with cardiac cachexia [7-9]. LFTs have previously been characterized in patients with chronic heart failure and correlated to outcomes [10]; However to date no information exists on the correlation of abnormal LFTs at the time of diagnosis of heart failure secondary to left ventricular systolic dysfunction (LVSD) in the outpatient setting (OP) to patient outcomes. The purpose of this study was to characterize LFTs in a cohort of patients newly diagnosed with HFREF in OP and to explore the impact they have on patient outcomes.

\section{Materials and Methods}

A retrospective analysis of all the patients diagnosed with heart

*Corresponding author: Prithwish Banerjee, Department of Cardiology, Honorary Professor of Cardiology, University Hospitals Coventry and Warwickshire, Coventry, England, UK, Tel: 00442476965670; Fax: 00442476965657; E-mail: Prithwish.Banerjee@uhcw.nhs.uk

Received October 27, 2016; Accepted November 20, 2017; Published November 23, 2017

Citation: Koganti S, Dasgupta S, Crossman R, Banerjee P (2017) Association of Abnormal Liver Function Tests to Outcomes in Patients with a New Diagnosis of Heart Failure with Reduced Ejection Fraction in the Outpatient Clinic. J Cardiovasc Dis Diagn 5: 300. doi: 10.4172/2329-9517.1000300

Copyright: ( 2017 Koganti S, et al. This is an open-access article distributed under the terms of the Creative Commons Attribution License, which permits unrestricted use, distribution, and reproduction in any medium, provided the original author and source are credited. 
Citation: Koganti S, Dasgupta S, Crossman R, Banerjee P (2017) Association of Abnormal Liver Function Tests to Outcomes in Patients with a New Diagnosis of Heart Failure with Reduced Ejection Fraction in the Outpatient Clinic. J Cardiovasc Dis Diagn 5: 300. doi: 10.4172/23299517.1000300

Page 2 of 4

failure at University Hospitals of Coventry and Warwickshire (UHCW) NHS Trust, England was carried out. We identified a group of patients with abnormal LFTs. Choosing randomly, a contemporaneous group with normal LFTs was also created as a comparator group. Both the groups were matched for age and sex. Only patients diagnosed with HFREF were included in the study. Patients with right heart failure or heart failure with preserved ejection fraction were not included. Patients with abnormal LFTs thought to be secondary to alcohol excess and medications were also excluded from the study. The four LFTs used in this study and their normal values are given in Table 1.

UHCW NHS Trust is a large tertiary cardiac center based in the county of West Midlands, UK providing multi-system specialist services to the local population. The patients attended a dedicated once a week consultant lead, one-stop, heart failure clinic held at the hospital with provision for echocardiography on arrival. Baseline blood tests including LFTs were performed on arrival. A comprehensive management plan along with input from a multidisciplinary team involving specialist nurses and a rehabilitation team was instituted on the same day of the patient being seen and diagnosed. Patients who required further investigations such as coronary angiography, cardiac magnetic resonance imaging and interventional procedures were referred on in an expedite manner. Thus, the source population for the study was from the heart failure clinic.

Baseline characteristics and baseline biochemistry measurements were recorded from the patient electronic record system. Echocardiogrpahic data relating to left ventricular (LV) ejection fraction (EF) and aetiology were obtained from patient records. Follow up data including death, hospitalization and length of stay were extracted from patient records.

\section{Statistical Methods}

Baseline continuous variables were described either using means with standard deviations or medians with interquartile ranges depending upon their distribution. Categorical variables were described using percentages. The multivariable modeling was created from the overall dataset using the generalized linear regression package in R. Both univariate and multivariate models were built to assess the statistical contribution of each variable. $\mathrm{P}<0.05$ was set as the level of significance. Kaplan-Meier survival curves were used to depict time to event data on death. All statistical analyses were performed using SPSS V.21.0 (SPSS Inc, Chicago, Illinois, USA).

\section{Results}

Between the years 2007 and 2011 we chose a total of 222 patients with HFREF for our study (as described in 'methods'). In the total cohort 153 (69\%) patients were male and 69 (31\%) were female. The cohort was divided into HFREF patients with abnormal LFT (95) and HFREF patients with normal LFT (127). Baseline clinical characteristics including laboratory measurements comparing the abnormal LFT group and the normal LFT group are shown in Table 2 . Three patients were excluded from the analysis as the data was missing. In this predominantly male cohort of white racial background coronary artery

\begin{tabular}{|c|c|}
\hline Liver function tests & Normal values \\
\hline ALT & $10 \mathrm{IU} / \mathrm{L}-40 \mathrm{IU} / \mathrm{L}$ \\
\hline ALP & $40 \mathrm{IU} / \mathrm{L}-130 \mathrm{IU} / \mathrm{L}$ \\
\hline Albumin & $35 \mathrm{~g} / \mathrm{dl}-50 \mathrm{~g} / \mathrm{dl}$ \\
\hline Bilirubin & $5 \mathrm{mg} / \mathrm{dl}-25 \mathrm{mg} / \mathrm{dl}$ \\
\hline
\end{tabular}

ALT: Alanine Aminotransferase; ALP: Alkaline Phosphatase

Table 1: LFTs with their normal values as used in the study.

\begin{tabular}{|c|c|c|c|}
\hline \multicolumn{5}{|c|}{ Characteristic } & Normal group (n=124) & Abnormal group (n=95) & p-value \\
\hline Age & $69.9 \pm 12.3$ & $68.6 \pm 14.3$ & 0.46 \\
\hline Gender (M) & $85(68.5 \%)$ & $66(69.5 \%)$ & NS \\
\hline Ethnicity (white) & $99(79.8 \%)$ & $74(77.8 \%)$ & NS \\
\hline NYHA 2 & $71(57.3 \%)$ & $50(52.6 \%)$ & NS \\
\hline NYHA3/4 & $33(26.7 \%)$ & $31(32.6 \%)$ & NS \\
\hline EF & $35.6 \pm 11.5$ & $36.7 \pm 13.8$ & 0.5 \\
\hline CAD & Medical History & & \\
\hline Hypertension & $73(42.7 \%)$ & $38(40 \%)$ & NS \\
\hline Diabetes mellitus & $32(25.8 \%)$ & $51(53.7 \%)$ & NS \\
\hline Atrial Fibrillation & $30(24.2 \%)$ & $21(22.1 \%)$ & NS \\
\hline Paced & $12(9.7 \%)$ & $34(35 \%)$ & NS \\
\hline Smoker & $44(35.5 \%)$ & $9(9.5 \%)$ & NS \\
\hline ACE-I & $77(62.1 \%)$ & $38(40 \%)$ & NS \\
\hline Betablockers & $64(51.6 \%)$ & $47(49.5 \%)$ & 0.06 \\
\hline Bilirubin total & $11.5 \pm 6.5$ & $31(32.6 \%)$ & 0.05 \\
\hline ALT & $21 \pm 8$ & $20.9 \pm 17$ & $<0.001$ \\
\hline ALP & $71 \pm 19.6$ & $108.8 \pm 327.6$ & 0.004 \\
\hline Albumin & $41 \pm 6$ & $37 \pm 6.8$ & $<0.001$ \\
\hline Sodium mmol/L & $137.2 \pm 9.5$ & $140 \pm 3.8$ & 0.06 \\
\hline Creatinine $(\mu m o l / /)$ & $116.9 \pm 83.9$ & $136.8 \pm 93.8$ & 0.1 \\
\hline
\end{tabular}

Table 2: Baseline characteristics and LFTs.

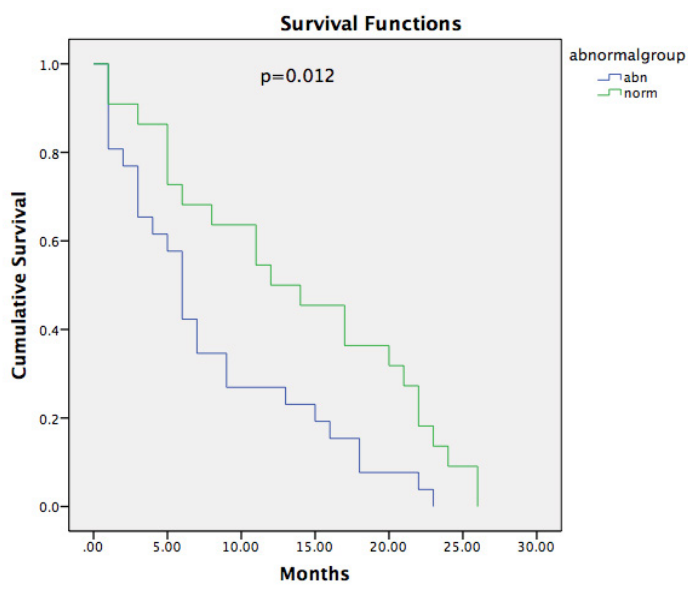

Figure 1: Kaplan-Meier survival curves.

disease (CAD) was the major etiological factor for the LVSD. There was no significant difference between the baseline characteristics (past medical history, functional class and LVEF) between the two groups. However, the use of beta-blockers was more prevalent in the normal LFT group (borderline statistical significance, $\mathrm{p}=0.05$ ). Looking at the abnormal LFT group, isolated elevation of ALT and of ALP was seen in 26 and 37 patients respectively. Isolated low Albumin was seen in 10 patients, but isolated Bilirubin elevation was not seen in this cohort. The rest had a combination of abnormal LFTs.

\section{Association of LFTs to Outcomes}

Once the diagnosis of HFREF was identified, baseline LFTs were recorded. We then documented outcomes for individual patients at one year. We looked for all-cause mortality, heart failure related admissions and length of stay. In total, across both the groups there were 48 events, which included 42 deaths. At 1 year, there were 26 events (23 deaths 
Citation: Koganti S, Dasgupta S, Crossman R, Banerjee P (2017) Association of Abnormal Liver Function Tests to Outcomes in Patients with a New Diagnosis of Heart Failure with Reduced Ejection Fraction in the Outpatient Clinic. J Cardiovasc Dis Diagn 5: 300. doi: 10.4172/23299517.1000300

Page 3 of 4

and 3 admissions related to heart failure) in the abnormal LFT group when compared to 22 events in the normal LFT group (19 deaths and 3 admissions), reaching statistical significance on time to event analysis using log rank test ( $\mathrm{p}=0.01$ ) (Figure 1). Below we discuss association of LFTs to different outcomes.

\section{Mortality}

Univariate analysis of LFTs on mortality revealed that both ALP and Albumin were statistically significant (OR 0.996; CI 0.001-0.999, $\mathrm{p}<0.01$ and OR 1.26; CI1.19-1.37 $\mathrm{p}<0.0001$ respectively). An increase of one unit of ALP imparted $0.4 \%$ increase of OR suggesting an increased chance of death. Likewise, one unit decrease in albumin increased the OR by $26 \%$. However, on multivariate analysis ALP was not significant with only albumin being significant (OR1.15; CI1.24-1.34, p<0.0001). Univariate and multivariate analysis repeated incorporating renal function along with LFTs showed that creatinine and albumin are strongly associated with mortality (OR 0.993; CI-0.986-0.998, $\mathrm{p}=0.017$ and 1.24; CI 1.15-1.35, $\mathrm{p}<0.001)$. The multivariate analysis on mortality of LFTs along with renal function is shown in Table 3.

\section{Admissions to Hospital}

Although ALT seemed to be statistically significant on univariate analysis this was not the case on multivariate modeling when LFTs and renal function were analyzed together.

\section{Length of Hospital Stay}

Patients in the group with abnormal LFTs stayed longer in the hospital following admissions $(25.9 \pm 29.1$ days vs. $17.2 \pm 20.5$ days, $\mathrm{p}=0.01$. On multivariate analysis of all LFTs and renal function (Table 4), Albumin appears to play a significant role (coefficient $-0.583, \mathrm{CI}$ -1.119 to $-0.046, \mathrm{p}=0.035$ ) and to an extent ALP (coefficient 0.033, CI $0.005,0.061, \mathrm{p}=0.02$ ). Every unit increase of ALP resulted in additional 0.045 days in hospital where as every unit increase of Albumin resulted in 0.875 days reduction in hospital stay.

\section{Discussion}

Our analysis clearly demonstrates the prognostic importance of LFTs on outcomes in patients with LVSD. It is evident that in patients with HFREF, the presence of abnormal LFTs resulted in more events

\begin{tabular}{|c|c|c|c|c|c|}
\hline $\begin{array}{c}\text { Liver function } \\
\text { tests }\end{array}$ & Coefficient & $\begin{array}{c}\text { Standard } \\
\text { error }\end{array}$ & P value & OR & OR 95\% Cl \\
\hline ALT & -0.001 & 0.002 & 0.556 & 0.999 & {$[0.994-1.00]$} \\
\hline ALP & $-3.14 \times 10^{-4}$ & 0.001 & 0.828 & 1 & {$[0.996-1.00]$} \\
\hline Albumin & 0.217 & 0.041 & $8.83 \times 10^{-8}$ & 1.24 & {$[1.15-1.35]$} \\
\hline Bilirubin & 0.009 & 0.011 & 0.429 & 1.01 & {$[0.980-1.03]$} \\
\hline Creatinine & -0.008 & 0.003 & 0.017 & 0.993 & {$[0.986-0.998]$} \\
\hline Sodium & -0.003 & 0.025 & 0.9 & 0.997 & {$[0.939-1.05]$} \\
\hline
\end{tabular}

Table 3: Multivariate analysis on mortality.

\begin{tabular}{|c|c|c|c|c|}
\hline $\begin{array}{c}\text { Liver function } \\
\text { tests }\end{array}$ & Coefficient & $\begin{array}{c}\text { Standard } \\
\text { error }\end{array}$ & P value & $\begin{array}{c}\text { Coefficient } \\
\mathbf{9 5 \%} \mathbf{~ C l}\end{array}$ \\
\hline ALT & 0.002 & 0.008 & 0.837 & {$[-0.014,0.017]$} \\
\hline ALP & 0.033 & 0.014 & 0.022 & {$[0.005,-0.061]$} \\
\hline Albumin & -0.583 & 0.274 & 0.035 & {$[-1.119,-0.046]$} \\
\hline Bilirubin & 0.006 & 0.095 & 0.953 & {$[-0.181,0.193]$} \\
\hline Creatinine & 0.02 & 0.021 & 0.346 & {$[-0.021,0.060]$} \\
\hline Sodium & -0.201 & 0.252 & 0.427 & {$[-0.695,0.294]$} \\
\hline
\end{tabular}

Table 4: Multivariate analysis on length of stay. Creatinine and sodium are included with the LFTs. The column showing $95 \% \mathrm{Cl}$ shows ranges separated by a comma if the range includes at least one negative value. and carried a poor prognosis at one year. The pathophysiological explanation for the abnormal LFTs may be congestive hepatopathy as well as low output states causing hepatopathy, both of which are part of LVSD. Moreover, it is possible that liver copes better to any gradual insult owing to its dual blood supply. Thus, abnormal LFTs in this cohort of patients may reflect chronic damage unlike the shock liver seen in acute hemodynamic collapse. For this reason, abnormal LFTs at the time of diagnosis or subsequently may warrant intensification of heart failure therapy. However, some caution is required as intensive heart failure therapy in itself can result in hypotensive states affecting the liver.

It is evident in our analysis that there was a trend towards higher usage of beta-blockers and ACE-I in the group with normal LFTs at the time of diagnosis of LVSD. It is plausible that this had a beneficial effect and LFTs were therefore less abnormal in this group when compared to the other group. The reason behind this disparity is not clear but the slightly increased prevalence of hypertension, diabetes and CAD in the normal LFT group could be one of the reasons.

Our analysis showed abnormal ALP and albumin to have a strong statistical association with death and length of hospital stay. This is different to previous studies, which have found some correlation between bilirubin with death [11-13].

We were not surprised to see patients with low albumin suffer more events as low albumin in heart failure is common and has been linked to a poor prognosis in several studies [14]. The possible causes of low albumin in heart failure include malnutrition, inflammation, cachexia, hemodilution, liver dysfunction, protein-losing enteropathy, increased transcapillary escape rate, and nephrotic syndrome [14,15]. Of these; inflammation, cachexia secondary to catabolic/anabolic imbalance, and nutritional deficiencies are probably the most important. One of the reasons behind poor nutrition in heart failure patients is oedema of the intestinal wall and resulting poor absorption of oral nutrients. This may respond to appropriate diuresis.

It was surprising to see elevated ALP levels in the absence of clinically overt congestive states in our study. Previous studies have shown cholestatic pattern of LFT abnormality in patients with biventricular failure, congestive cardiac failure or in valvular abnormalities such as tricuspid regurgitation [16]. This can be explained by hepatic congestion due to increased venous pressure resulting in cholestasis and elevated ALP and Bilirubin. However elevated cholestatic enzymes in the absence of right heart involvement is puzzling although this was noted in the study carried out by Poelzl et al. [17]. In their cohort consisting of 1032 patients with chronic heart failure the predominant abnormality was elevation in cholestatic enzymes. They proposed a cardio-hepatic syndrome model as the potential explanation for elevation in cholestatic enzymes; similar to the cardio-renal syndrome [18].

LFTs were previously characterized in the acute decompensated heart failure setting [19] but to date no association of prognosis with LFTs at the time of diagnosis has been carried out. Somewhat similar findings to our cohort were noted during post-hoc analysis of EVEREST trial [20]; however, that was carried out in the setting of hospitalized patients where as in our cohort all the patients were out patient based.

\section{Conclusion}

The presence of abnormal LFTs, at the time of diagnosis of HFREF in the outpatient clinic, seems to be associated with a worse outcome. Low albumin in patients with heart failure needs particular attention, as aggressive management and close monitoring are required along 
Citation: Koganti S, Dasgupta S, Crossman R, Banerjee P (2017) Association of Abnormal Liver Function Tests to Outcomes in Patients with a New Diagnosis of Heart Failure with Reduced Ejection Fraction in the Outpatient Clinic. J Cardiovasc Dis Diagn 5: 300. doi: 10.4172/23299517.1000300

with a nutritional assessment. Further studies are required to look into the explanation for cholestatic pattern of LFT abnormalities in left ventricular systolic dysfunction and the feasibility of including LFTs into risk scoring models to help in prognostication.

\section{Limitations}

This study is a retrospective analysis on a relatively small number of patients from an existing database of a large single tertiary cardiac center and the limitations associated with such analysis need recognition. However, the uniqueness of our study is the characterization of LFTs at the time of diagnosis of heart failure in an outpatient setting.

\section{Acknowledgements}

We would like to acknowledge Ann Nugent for some of the initial statistical input.

\section{References}

1. Petersen SRM, Wolstenholme J (2002) Coronary heart disease statistics: Heart failure supplement London: British Heart Foundation.

2. Cowie MR, Wood DA, Coats AJ, Thompson SG, Poole-Wilson PA, et al. (1999) Incidence and aetiology of heart failure; a population-based study. Euro Heart J 20: 421-428.

3. Owan TE, Hodge DO, Herges RM, Jacobsen SJ, Roger VL, et al. (2006) Trends in prevalence and outcome of heart failure with preserved ejection fraction. $\mathrm{N}$ Engl J Med 355: 251-259

4. Hobbs FD, Roalfe AK, Davis RC, Davies MK, Hare R, et al. (2007) Prognosis of all-cause heart failure and borderline left ventricular systolic dysfunction: 5-year mortality follow-up of the Echocardiographic Heart of England Screening Study (ECHOES). Eur Heart J 28: 1128-1134.

5. Lau GT, Tan HC, Kritharides L (2002) Type of liver dysfunction in heart failure and its relation to the severity of tricuspid regurgitation. Am J Cardiol 90: 14051409.

6. Seeto RK, Fenn B, Rockey DC (2000) Ischemic hepatitis: Clinical presentation and pathogenesis. Am J Med109: 109-113.

7. Arques $S$ (2011) Hypoalbuminemia as a contributor to the progression of cardiovascular diseases. Ann Thorac Surg 92: 2305.

8. Arques S, Ambrosi P (2011) Human serum albumin in the clinical syndrome of heart failure. J Cardiac Fail 17: 451-458.

9. Arques S, Roux E, Stolidi P, Gelisse R, Ambrosi P (2011) Usefulness of serum albumin and serum total cholesterol in the prediction of hospital death in older patients with severe, acute heart failure. Arch Cardiovascular Dis 104: 502-508.

10. Batin P, Wickens M, McEntegart D, Fullwood L, Cowley AJ (1995) The importance of abnormalities of liver function tests in predicting mortality in chronic heart failure. Eur Heart J 16: 1613-1618.

11. Chintanaboina J, Haner MS, Sethi A, Patel N, Tanyous W, et al. (2013) Serum bilirubin as a prognostic marker in patients with acute decompensated heart failure. Korean J Int Med 28: 300-305

12. Allen LA, Felker GM, Pocock S, McMurray JJ, Pfeffer MA, et al. (2009) Liver function abnormalities and outcome in patients with chronic heart failure: data from the Candesartan in Heart Failure: Assessment of Reduction in Mortality and Morbidity (CHARM) program. Euro J Heart Fail 11: 170-177.

13. Shinagawa $H$, Inomata $T$, Koitabashi T, Nakano H, Takeuchi I, et al. (2008) Prognostic significance of increased serum bilirubin levels coincident with cardiac decompensation in chronic heart failure. Circulation J 72: 364-369.

14. Horwich TB, Kalantar-Zadeh K, MacLellan RW, Fonarow GC (2008) Albumin levels predict survival in patients with systolic heart failure. Am Heart $\mathrm{J} 55$ : 883-889.

15. Sandek A, Doehner W, Anker SD, Von Haehling S (2009) Nutrition in heart failure: an update. Curr Opin Clin Nutr Metab Care 12: 384-391.

16. Vyskocilova K, Spinarova L, Spinar J, Mikusova T, Vitovec J, et al. (2014) Prevalence and clinical significance of liver function abnormalities in patients with acute heart failure. Biomedical Papers.

17. Poelzl G, Ess M, Mussner-Seeber C, Pachinger O, Frick M, et al. (2012) Liver dysfunction in chronic heart failure: Prevalence, characteristics and prognostic significance. Euro J Clin Invest 42: 153-163.

18. Poelzl G, Auer J (2014) Cardiohepatic syndrome. Curr Heart Failure Reports 12: $68-78$

19. Nikolaou M, Parissis J, Yilmaz MB, Seronde MF, Kivikko M, et al. Liver function abnormalities, clinical profile, and outcome in acute decompensated heart failure. Eur Heart J 34: 742-749.

20. Ambrosy AP, Vaduganathan M, Huffman MD, Khan S, Kwasny MJ, et al. (2012) Clinical course and predictive value of liver function tests in patients hospitalized for worsening heart failure with reduced ejection fraction: an analysis of the EVEREST trial. Euro J Heart Failure 14: 302-311. 